\title{
BMJ Open Associations between cardiometabolic risks and physical activity in early adulthood: a cross-sectional study
}

\author{
Hsin-Yen Yen (D) , ${ }^{1}$ Ching Li ${ }^{2}$
}

To cite: Yen H-Y, Li C. Associations between cardiometabolic risks and physical activity in early adulthood: a crosssectional study. BMJ Open 2021;11:e042668. doi:10.1136/ bmjopen-2020-042668

- Prepublication history for this paper is available online. To view these files, please visit the journal online (http://dx.doi. org/10.1136/bmjopen-2020042668).

Received 10 July 2020 Revised 15 April 2021 Accepted 20 April 2021

\section{Check for updates}

\section{(c) Author(s) (or their} employer(s)) 2021. Re-use permitted under CC BY-NC. No commercial re-use. See rights and permissions. Published by BMJ.

${ }^{1}$ School of Gerontology Health Management, College of Nursing, Taipei Medical University, Taipei, Taiwan ${ }^{2}$ Graduate Institute of Sport, Leisure and Hospitality Management, National Taiwan Normal University, Taipei, Taiwan

Correspondence to

Dr Ching Li;

t94002@ntnu.edu.tw

\section{ABSTRACT}

Objectives Young adults' physical activity $(\mathrm{PA})$ is a foundation of creating future healthy lifestyles. The purpose of this study was to explore differences in PA, sedentary behaviour, walkability and health beliefs among young adults with different levels of cardiometabolic risks and the influence of moderate-to-vigorous PA.

Design A cross-sectional study was conducted using a structured questionnaire.

Participants Totally, 1149 valid responses were received for a response rate of $86.32 \%$. According to the self-reported worst health condition, participants were categorised into healthy adults, and adults at levels 1 (overweight), 2 (obese), 3 (hypertensive, hyperlipidaemic, and/or hyperglycaemic), and 4 (with a diagnosis of cardiometabolic diseases) of cardiometabolic risks. Primary outcome measures PA, sedentary time, walkability and health beliefs.

Results Significant differences in PA $(F=3.78, p<0.01)$ and sedentary time $(F=2.39, \mathrm{p}<0.05)$ among groups with various cardiometabolic risk levels were found. Healthy adults and adults at level 1 risk had significantly higher PA than adults at risk levels 2 and 3 . Young adults at risk level 3 were less likely to participate in moderate-to-vigorous PA than were healthy adults $(\mathrm{OR}=0.64(95 \% \mathrm{Cl} 0.41$ to 0.99$), p<0.05)$. Individuals who perceived that there were more recreational facilities $(0 R=1.27$ (1.05 to 1.53), $\mathrm{p}<0.05)$, who had higher benefits of exercise $(0 \mathrm{R}=1.73$ (1.30 to 2.31), $p<0.001)$, and who had lower barriers to exercise ( $\mathrm{OR}=0.42$ ( 0.32 to 0.55$), \mathrm{p}<0.001$ ) were more likely to participate in moderate-to-vigorous PA.

Conclusions Being aware of body weight changes could be a danger sign of a lack of PA. Developing environmental and psychological strategies to promote engaging in PA is necessary to promote the cardiometabolic health of young adults.

\section{INTRODUCTION}

Physical inactivity is the fourth leading cause of death. Individuals who spend lots of time engaged in sedentary behaviours have increased risks of non-communicable diseases (NCDs), such as obesity, cardiovascular diseases, type II diabetes and certain cancers. ${ }^{12}$ Every year $63 \%$ of deaths are estimated to result from NCDs worldwide. ${ }^{3}$

Physical activity (PA) is one of the most effective modifiable behaviours in order

\section{Strengths and limitations of this study}

Young adults were categorised into five levels of cardiometabolic risks from level 0 (healthy adults) to level 4 (with a diagnosis of cardiometabolic disease).

- In total, 1331 participants responded, for a valid response rate of $86.32 \%$.

- The power of the final model was $16.0 \%$ for predicting moderate-to-vigorous physical activity.

- This cross-sectional study can present associations but cannot present cause-and-effect relationships.

- An internet survey has potential sampling and self-reported recall biases and possibly may lack representativeness.

to prevent NCDs, especially cardiometabolic diseases (ie, hypertension, hyperlipidaemia, diabetes and metabolic syndrome). Increasing moderate-to-vigorous PA (MVPA) and decreasing sedentary behaviours are key to preventing NCDs and promoting health. Consistently, integrating MVPA into one's daily life leads to lower prevalence rates and mortality due to cardiometabolic diseases. ${ }^{4-6}$ Engaging in MVPA and exercise training improves cardiopulmonary function, physical fitness, insulin sensitivity and muscle power, while decreasing the body mass index (BMI). ${ }^{78}$ However, even though PA are important in preventing NCDs, $21.4 \%$ of people worldwide still engage in an inactive daily lifestyle. ${ }^{9}$

If a decline in PA occurs, the first priority is to identify personal characteristics, the physical environment, and psychological factors. ${ }^{10}$ Two main strategies for promoting individuals' MVPA are environmental and psychological. ${ }^{11}$ Characteristics of the living environment influence residents' behaviour, especially in terms of walkability. ${ }^{12}$ Creating a walkable environment is beneficial for pedestrians to increase walking behaviours and recreational $\mathrm{PA}$, with such benefits as friendly neighbours, short distances to recreational facilities, aesthetic views, accessible 
infrastructure and a feeling of safety. ${ }^{13}{ }^{14}$ Walkability is associated with people's PA and sedentary behaviours. ${ }^{15}$ People living in an environment with higher walkability might have higher frequencies and longer time of PA. ${ }^{16}$ Therefore, high walkability also brings about benefits of lower incidence rates of cardiometabolic diseases through the mediator of MVPA. ${ }^{17}$

On the other hand, PA is associated with intrapersonal and interpersonal factors in psychological domains, including socioeconomics, knowledge, attitudes and social interactions. ${ }^{1819}$ The Health Beliefs Model is often used to explain the psychological domain of health behaviour, for example, health beliefs of PA (HBPA) ${ }^{19}$ Once people are aware of the serious consequences of physical inactivity, they might begin to achieve minimal PA requirements. When perceived benefits are higher than the costs of PA, people will choose an active lifestyle. In contrast, individuals with insufficient knowledge of future health threats of physical inactivity or who perceive many barriers to exercise might spend much time in sedentary behaviours. ${ }^{20}$ Social relationships with family, friends and neighbours also impact PA self-choices. ${ }^{21}$

The prevalence of engaging in PA in healthy populations also differs from that of populations with chronic conditions. ${ }^{22}$ One meta-analysis that compared healthy children and adolescents to those with chronic diseases found that patients with cardiovascular diseases or diabetes had lower amounts of PA per day, fewer days when they achieved the MVPA standard, and longer sedentary time than the healthy control group. ${ }^{23}$ Healthy older adults have a higher level of leisure-time PA and shorter sedentary time than older adults with two and more diagnoses of chronic diseases. ${ }^{24}$ However, few studies have focused on comparisons of young adults as the main target population.

In Erikson's stages of human psychological development, individuals at the ages of 20-39 years are in early adulthood. ${ }^{25}$ Young adults, especially those aged 18-45 years, experience many major life changes, such as graduation and marriage. Such life changes impact one's selfidentity, self-determination, future lifestyle and long-term health behaviours. ${ }^{26}$ However, few studies have discussed the relationship of young adults' health behaviours and cardiometabolic diseases. In this population, it is important to identify environmental and psychological risk factors that affect changes in health behaviours.

Young adults' lifestyle and health behaviours form the foundation of creating a lifestyle when they are older. Therefore, the purpose of this study was to explore differences in PA, walkability and health beliefs between healthy young adults and young adults with different levels of cardiometabolic risks, and determine the influence of cardiometabolic risks, walkability and health beliefs on MVPA. The research questions were as follows:

1. Are there any differences in PA, sedentary behaviour, perceived walkability and health beliefs in young adults with different levels of cardiometabolic risk?
2. Are cardiometabolic risks, perceived walkability and health beliefs associated with MVPA?

\section{METHODS}

\section{Participants and sampling}

A cross-sectional study was conducted from December 2016 to June 2017. The target population was urban residents in early adulthood in Taiwan. Therefore, an internet survey through social media was conducted using Facebook, Instagram, Twitter and various forums. The inclusion criteria of participants were (1) young adults aged 18-45 years, (2) currently living in an urban area and (3) with no physical or mental disability. Participants were required to complete an anonymous questionnaire and consent to participate in the survey.

In the sampling process, the Walkability Index (WI) in each administrative region was calculated by the sum of Z-scores of street connectivity, land use mix, residential density, socioeconomic status and crime rate from objective open government data. ${ }^{27}{ }^{28}$ According to the WI, 19 administrative regions in Taiwan were ranked into four levels. Quota sampling was used to recruit 300 participants from each level based on their current address. In total, 1331 people responded, and the valid response rate was $86.32 \%$ after excluding participants who did not meet the inclusion criteria. This number of participants satisfied the minimal sample size $(n>384)$ for a population exceeding 10000 for internet survey research.

\section{Patient and public involvement}

Patients or the public were not involved in the design, or conduct, or reporting, or dissemination plans of our research.

\section{Data collection}

All outcomes were measured using self-reported data. Participants were required to complete a structured questionnaire composed of four parts to collect data. The first part was their demographic background, including questions of gender, age, educational level (college: associate and bachelor's degree; graduate school: master's and doctoral degree; others), and income. The self-reported current health condition concerned participant's height, weight and two items of cardiometabolic risks. The first item was 'Have you ever found that you have the following health conditions (hypertension, hyperlipidaemia, and/ or hyperglycaemic)?' The second item was 'Have you been diagnosed by a doctor to have the following chronic diseases and need treatment or long-term follow-up? (metabolic syndrome, hypertensive diseases, hyperlipidaemia, or type II diabetes)'.

The Physical Activity Neighborhood Environment Survey (PANES) ${ }^{29}$ was developed to evaluate perceived walkability in neighbourhood environments (walking time of 10-15 min). The inventory includes 17 items with a 4-point Likert scale. According to the scoring guide, ${ }^{30}$ the score was categorised into several constructs of walkability in a neighbourhood, such as land use mix, safety, 
infrastructure, recreational facilities, aesthetics, and so on. The higher the score, the higher the perceived walkability. Cronbach's alpha was 0.72 in this study.

The HBPA $^{31}$ was developed to measure psychological properties of PA according to the Health Beliefs Model. The HBPA inventory includes 41 items with a 5-point Likert scale. An exploratory factor analysis divided the inventory into five factors (Kaiser-Meyer-Olkin=0.92), including susceptibility to health problems, benefits of exercise, barriers to exercise, significant others' support, and cues to action. These factors were the same as those of the original HBPA inventory. The higher the score, the higher the perceived health beliefs. Cronbach's alpha was 0.92 in this study.

The International Physical Activity Questionnaire (IPAQ) collected participants self-reported PA over the previous 7 days. Participants' PA level (metabolic equivalents of task (MET)-min/week) was calculated in accordance with the scoring protocol. The higher the score, the higher the PA and sedentary time. Participants were also categorised as to whether or not they participated in MVPA, depending on whether their PA met the minimal requirement of MVPA (600 MET-min/week). ${ }^{32}$

\section{Data analysis}

According to their self-identified current health condition, participants were categorised into one of five levels of cardiometabolic risks. If an individual had more than one health condition of interest, the individual was given a higher level. For example, a participant with a simultaneous BMI of $\geq 27 \mathrm{~kg} / \mathrm{m}^{2}$, self-reported hypertension, and type II diabetes would be categorised in to level 4 of cardiometabolic risks.

Level 0: healthy young adults with no cardiometabolic risks;

Level 1: young adults with a BMI of 24-27 kg/ $\mathrm{m}^{2}$;

Level 2: young adults with a BMI of $\geq 27 \mathrm{~kg} / \mathrm{m}^{2}$;

Level 3: young adults who reported hypertension, hyperlipidemia, or hyperglycaemic;

Level 4: young adults who reported at least one diagnosis of metabolic syndrome, hypertensive diseases, hyperlipidemia, or type II diabetes.

Descriptive analyses of all variables were by the mean and SD. A $\chi^{2}$ test was used to identify associations between demographic background variables and levels of cardiometabolic risks. A one-way analysis of covariance (ANCOVA) with a post-hoc test was used to analyse differences in PA, sedentary behaviour, walkability and health beliefs among adults with different levels of cardiometabolic risks while using covariables of the demographic background. The variance inflation factor of variables ranged 1.05-2.40 without multicollinearity. OR estimates and $95 \%$ CIs of demographic background variables, cardiometabolic risks, walkability and health beliefs were obtained from a logistic regression model for predicting whether subjects participated in MVPA. The model included dummy variables for each level of cardiometabolic risk in reference to healthy adults. Nagelkerke $R^{2}$ was used as the explanatory power in the logistic regression model.

\section{RESULTS}

Demographic background variables and cardiometabolic risks Details of participants' demographic background are given in table 1 . Numbers in the five groups were 713 $(62.10 \%)$ healthy adults, $113(11.60 \%)$ with level 1 cardiometabolic risks, $68(5.9 \%)$ with level 2 cardiometabolic risks, $150(13.10 \%)$ with level 3 cardiometabolic risks, and $85(7.40 \%)$ with level 4 cardiometabolic risks. Significant associations were found between the frequencies of adults with different levels of cardiometabolic risks and gender $(\mathrm{p}<0.01)$, age $(\mathrm{p}<0.01)$, educational level $(p<0.01)$, and income $(p<0.01)$. Young adults with level 4 cardiometabolic risks were more frequently male $(67.06 \%)$, aged $26-45$ years $(44.70 \%)$, with a graduate degree $(62.35 \%)$, and with an annual income of New Taiwan US\$260 000-500 000 ( $\approx$ US\$8700-17 000) $(31.76 \%)$.

\section{Differences in PA and sedentary behaviours among participants with various cardiometabolic risks}

Results of the one-way ANCOVA and post-hoc tests are given in table 2. There was a significant effect of cardiometabolic risks on PA $(F=3.78, \mathrm{p}<0.01)$ and sedentary time $(F=2.39, \mathrm{p}<0.05)$ after controlling for gender, age, educational level and income. Post-hoc tests showed that healthy adults and adults at level 1 had significantly higher PA than adults at levels 2 and 3. Post-hoc tests also showed that healthy adults and adults at level 4 had significantly longer sedentary time than the group at level 2.

\section{Differences in walkability and health beliefs among those with cardiometabolic risks}

Tests of walkability and health beliefs were conducted using a one-way ANCOVA to compare the five groups with and those without cardiometabolic risk while controlling for gender, age, educational level and income (table 2). For environmental factors, only the residential density was found to significantly differ among young adults with different levels of cardiometabolic risks. Adults at level 4 resided in the lowest density residential areas. The effects of other indicators of walkability were not statistically significant among the different levels of cardiometabolic risks.

As to psychological factors, there were significant differences in susceptibility to health problems $(F=4.87$, $\mathrm{p}<0.01)$ and exercise barriers $(F=4.90, \mathrm{p}<0.01)$. Post-hoc tests revealed that the susceptibility to health problems in healthy adults and adults at level 1 was significantly lower than that in adults at levels 2 and 4. Exercise barriers in healthy adults and the group at level 1 were significantly lower than those of groups at levels 2, 3, and 4 . The results showed that effects of other indicators of health beliefs 


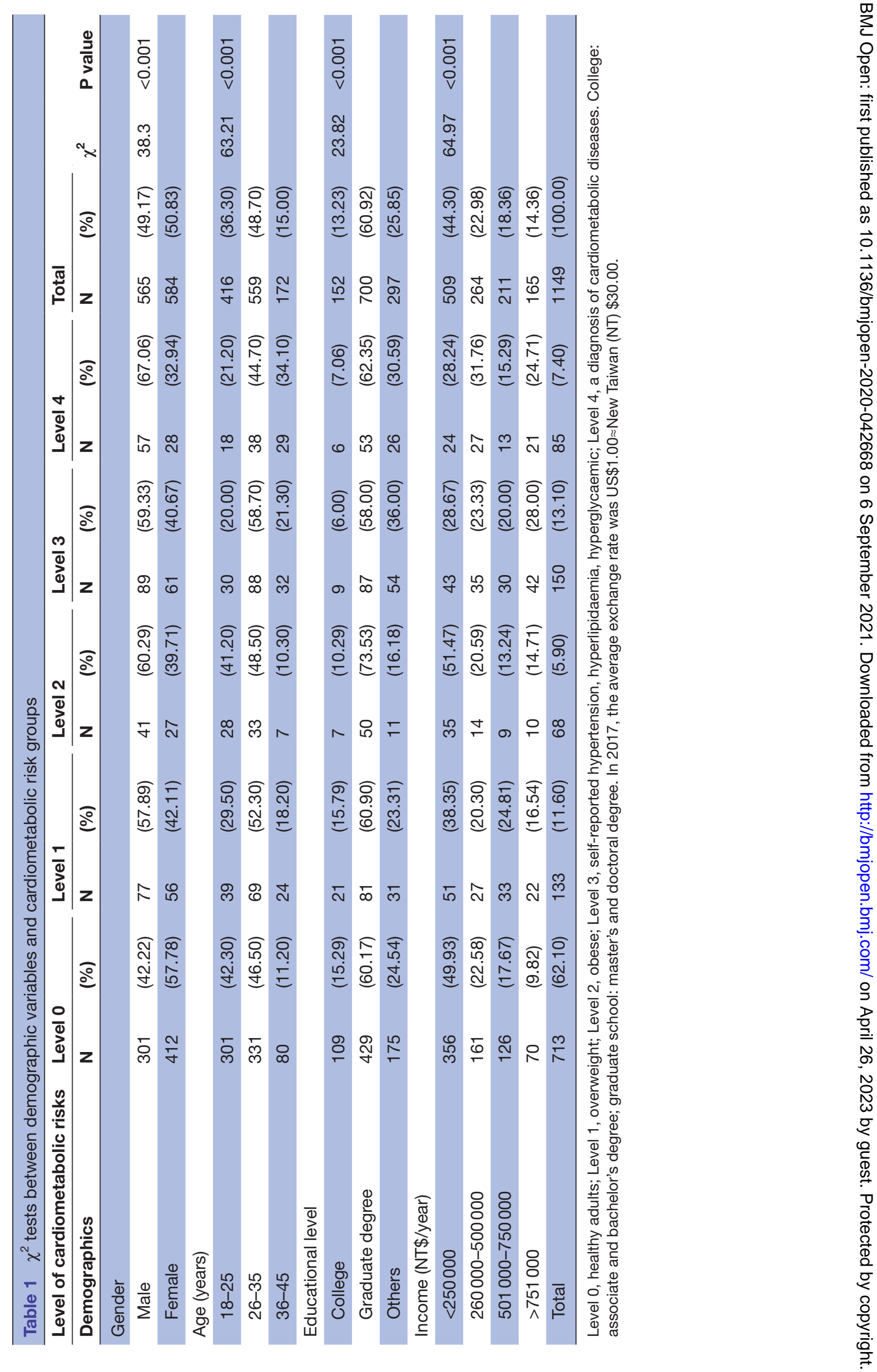




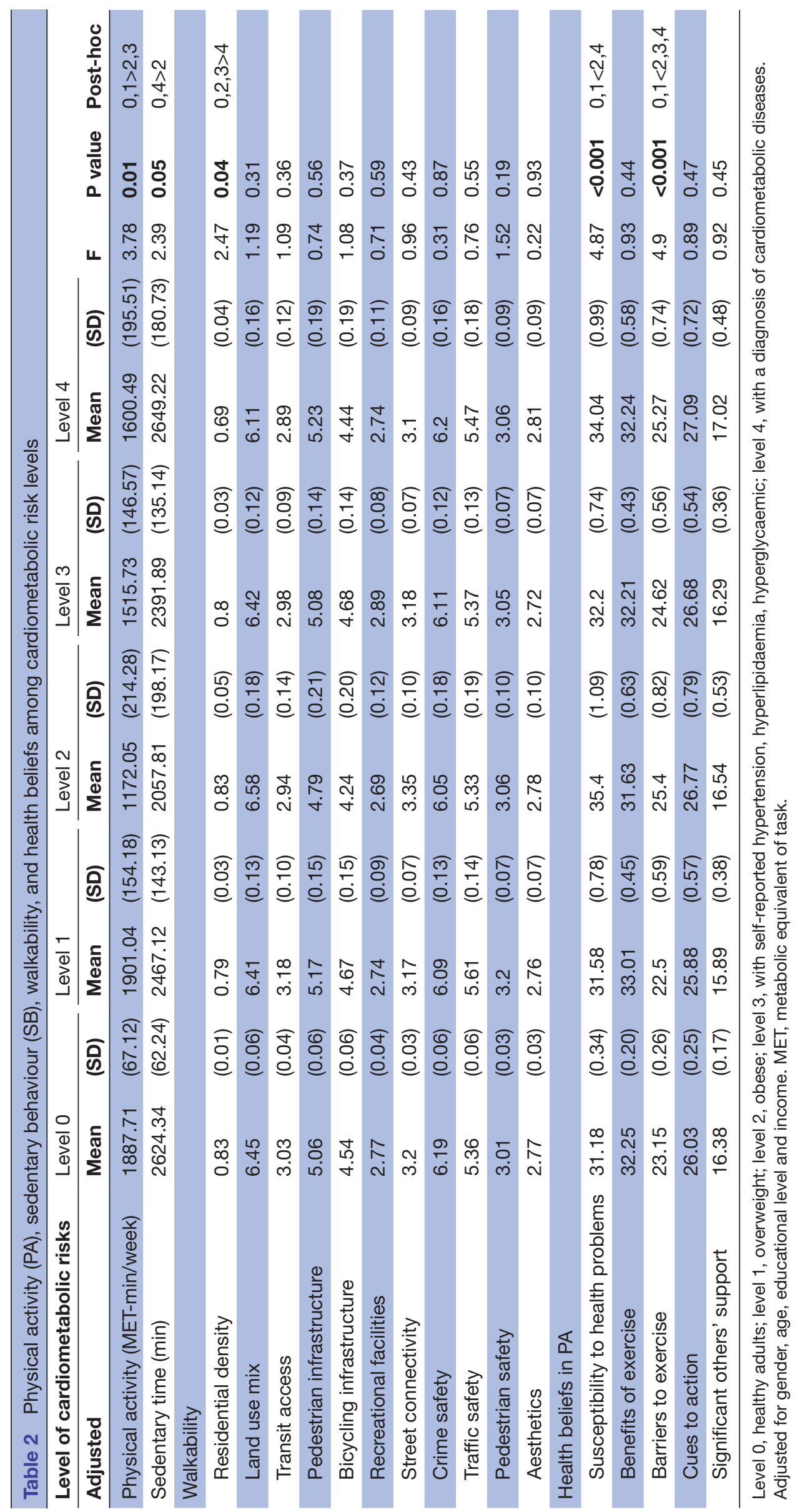


were no statistically significant, including exercise benefits, cues to action and significant others' support.

\section{Factors of MVPA}

The logistic regression results are given in table 3 . In model 1 , there were significant associations of gender, age, education and cardiometabolic risks with participation in MVPA. Females, older individuals, and those with a lower educational level were less likely to participate in MVPA. There was no significant association with income. Young adults at level 3 had a lower probability of engaging in MVPA than those in the healthy adult group (OR: 0.64 (95\% CI 0.41 to 0.99), $\mathrm{p}<0.05$ ). Demographic background and levels of cardiometabolic risks in the logistic model together accounted for $4.56 \%$ of the explanation for the probability of engaging in MVPA.

Model 2 added environmental factors, and this increased the explanatory power of the logistic model to $6.54 \%$. Only one factor of recreational facilities (OR: 1.27 (1.05 to 1.53$), \mathrm{p}<0.05$ ) had a significant association with MVPA. Individuals who perceived more recreation facilities in a walkable neighbourhood were more likely to participate in MVPA. There were no significant associations with other environmental factors.

Model 3 added psychological factors, and this increased the explanatory power to $15.96 \%$ as the final model. Two factor scores had significant associations with MVPA: benefits of exercise (OR: 1.73 (1.30 to 2.31), p<0.001) and barriers to exercise (OR: 0.42 (0.32 to 0.55$), \mathrm{p}<0.001)$. Individuals who understood the benefits of exercise and who perceived low barriers to exercise were more likely to participate in MVPA. No significant associations of susceptibility to health problems, cues to action, or significant others' support were observed in this model.

\section{DISCUSSION}

This study focused on adults in early adulthood. The present study found differences in demographic variables, PA and sedentary time among young adults at different levels of cardiometabolic risks. Differences in environmental and psychological factors of perceived residential density, susceptibility to health problems and barriers to exercise were also found among young adults with different levels of cardiometabolic risks. Finally, young adults with level 3 cardiometabolic risks were less likely to engage in MVPA. Recreational facilities, benefits of exercise and barriers to exercise were factors affecting MVPA engagement.

This study found that healthy young adults and young adults at level 1 had higher PA than those with higher levels of cardiometabolic risks. A difference in PA was found between levels $0-1$ and 2-3. Most previous studies also showed that healthy control groups had a longer duration and a higher level of PA and more days that they achieved MVPA recommendations than groups with cardiometabolic diseases. ${ }^{23}$ The PA level was negatively associated with an individual's number of diagnoses of chronic diseases. ${ }^{33}$ Therefore, it is important to be aware of changes in body weight which could be a danger sign of a lack of PA. However, the present study did not find a difference in PA between healthy adults and those at level 4. Chronic diseases can also be also a trigger of changes in $\mathrm{PA}^{34}$

The study also found that healthy young adults and those at level 4 had longer sedentary time than other risk groups. Previous studies presented no marked differences and did not reach a conclusion about sedentary time between healthy groups and groups with chronic diseases. ${ }^{23}{ }^{34}$ Healthy young adults have lifestyles with simultaneous high PA and long sedentary time. At this age, there is a balance between PA and sedentary behaviour. But it is possible to develop cardiometabolic risks when lifestyle changes cause an imbalance. ${ }^{4}$ Having cardiometabolic diseases and an inactive lifestyle could threaten the health and future life of young adults. Decreasing sedentary behaviours and increasing PA are both important in delaying pathological processes of current diseases.

Regarding environmental differences among young adult groups, only one indicator of walkability exhibited a significant difference. Young adults at level 4 had the lowest score of perceived residential density. Individuals with cardiometabolic diseases had different insights as to their neighbourhood environment. ${ }^{35}$ Walkability is a modifiable neighbourhood feature which can promote PA. Overall, PA is an important mediator between the environment and chronic illnesses. ${ }^{14}$ Regarding psychological differences among young adults, there were two domains in the HBPA with significant differences. Young adults at levels 2, 3 and 4 were more susceptible to health problems and barriers to exercise than were healthy young adults and those at level 1. A person's health and illness status impacted their health beliefs, especially those with chronic diseases. Patients with chronic diseases are more susceptible to health problem than are healthy adults. ${ }^{1136}$ Interestingly, a difference in the HBPA was also found between levels $0-1$ and 2-4.

The second main purpose of the present study was to determine the influence of cardiometabolic risks, walkability and health beliefs on MVPA. Young adults at level 3 were less likely to participate in MVPA. This study found that gender, age and educational level were associated with MVPA. Previous studies also showed that personal demographic variables, such as age, gender, race and years of education, were associated with chronic risk factors and disease in population-based studies. ${ }^{33}$ However, the predictive power of model 1 was low, so that environmental and psychological factors should be considered in the logistic regression model for predicting MVPA. Walkability was associated with residents' leisure-time PA, transportation choices and active lifestyles. ${ }^{37}$ In model 2 , only recreational facilities were a significant predictor of MVPA. The accessibility, distance, density and use of recreation facilities had positive associations with the level of PA in residents. ${ }^{38}$ A previous study also found that not every indicator of walkability had a direct association 


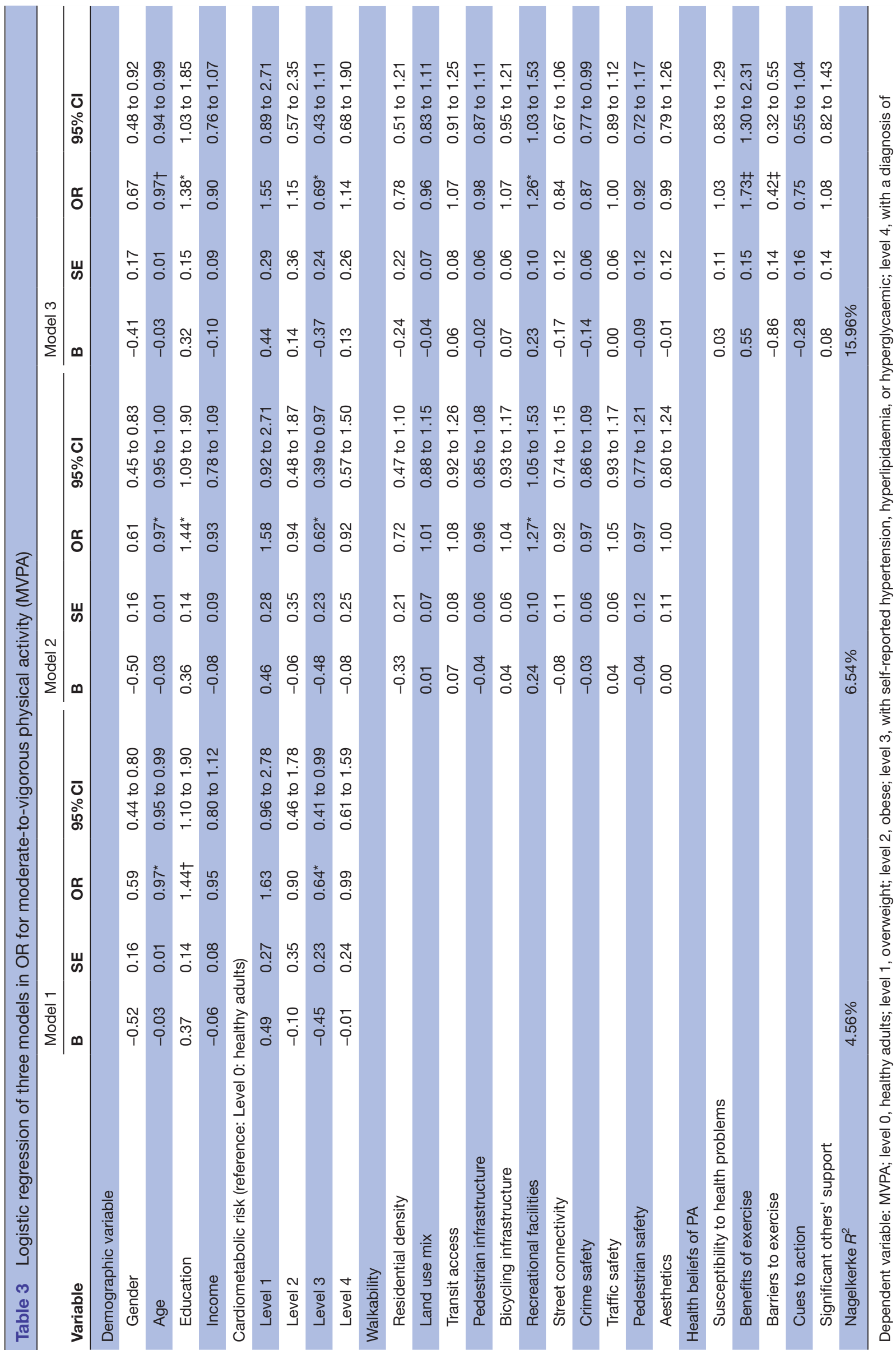


with PA or the risk of cardiometabolic diseases. ${ }^{39}$ In model 3, the benefits of exercise and barriers to exercise were important factors in MVPA engagement. Benefits of exercise were positively associated with MVPA. In contrast, barriers to exercise were negatively associated with MVPA. ${ }^{36} \mathrm{HBPA}$ is a motivation to increase PA participation. Previous studies also showed that indicators of health beliefs significantly predicted the odds of MVPA or achievement of minimal PA requirements. ${ }^{11} 40$ Once individuals perceive more benefits of than barriers to exercise, they are more likely to engage in MVPA. ${ }^{41}$

When indicators of HBPA were added to the model of demographic variables and walkability, the explanatory power increased $9.5 \%$ to reach $16 \%$. Young adults' thoughts were associated with their PA. Therefore, it is important to increase MVPA beginning with health education for young adults. Young adults' lifestyles are not settled and can easily be modified. Increasing knowledge and awareness of diseases can help young adults understand the benefits of PA and overcome barriers to exercise. MVPA can become a part of one's lifestyle that is beneficial for preventing cardiometabolic diseases and promoting health in their future lives.

There are some limitations in this study. First, this was a cross-sectional study which only focused on PA and cardiometabolic groups. The associations could not represent cause-and-effect relationships between variables. Second, the internet survey using structured questionnaires we conducted has potential for sampling and self-reported recall biases. All citizens were welcomed to complete the internet survey. The sample might have lacked representativeness of the population. Third, only $37.9 \%$ of participants had cardiometabolic risks, so small sample sizes of groups were used to compare differences. Also, participants were categorised into levels 2 and 3 because the BMI can be monitored simply at home by participants themselves, but hypertension, hyperlipidaemia and hyperglycaemia can only be measured by specific instruments. However, levels 2 and 3 were both cardiometabolic risk factors in previous studies. Finally, the explanatory power of the final model was still not high $(16.0 \%)$. There are still other factors that influence PA, such as other diseases, an unhealthy lifestyle, immigration, healthcare costs, community settings, air pollution and public policies, ${ }^{42}$ which should be taken into consideration in future studies.

\section{CONCLUSIONS}

This was a cross-sectional study that focused on associations of adults' PA with cardiometabolic risks in early adulthood. Results found higher PA and lower sedentary time in healthy adults than those with cardiometabolic risks. A difference in PA was found between levels 0-1 and 2-3, and between participants who were overweight and those who were obese. Level 3 cardiometabolic risks, perceived recreational facilities, benefits of exercise, and barriers to exercise were associated with participation in
MVPA. The present study provides information on young adults' PA to which public health practitioners, health educators and the government should pay greater attention. By overcoming barriers against PA and decreasing environmental disparities, young adults will have more opportunities to engage in PA. Shaping an active lifestyle is important for young adults' future well-being.

Contributors HYY: data curation; methodology; software; validation; formal analysis; visualisation; and writing. CL: conceptualisation; writing; project administration; and funding.

Funding This work was supported by the Ministry of Science and Technology, Taiwan under grant no. [NSC-105-2410-H-003-049].

\section{Competing interests None declared.}

Patient and public involvement Patients and/or the public were not involved in the design, or conduct, or reporting, or dissemination plans of this research.

Patient consent for publication Not required.

Ethics approval Ethical approval was granted by the Research Ethics Committee of National Taiwan Normal University (NTNU-REC 201605HM025).

Provenance and peer review Not commissioned; externally peer reviewed.

Data availability statement Data are available upon reasonable request.

Open access This is an open access article distributed in accordance with the Creative Commons Attribution Non Commercial (CC BY-NC 4.0) license, which permits others to distribute, remix, adapt, build upon this work non-commercially, and license their derivative works on different terms, provided the original work is properly cited, appropriate credit is given, any changes made indicated, and the use is non-commercial. See: http://creativecommons.org/licenses/by-nc/4.0/.

ORCID iD

Hsin-Yen Yen http://orcid.org/0000-0002-9194-1783

\section{REFERENCES}

1 Huang $\mathrm{X}$, Yang $\mathrm{H}$, Wang HHX, et al. The association between physical activity, mental status, and social and family support with five major non-communicable chronic diseases among elderly people: a cross-sectional study of a rural population in southern China. Int J Environ Res Public Health 2015;12:13209-23.

2 Dishman RK, Heath GW, Lee I-M. Physical activity epidemiology. 2nd ed. Champaign, Illinois: Human Kinetics, 2013. http://ezproxy. ithaca.edu:2048/login?url=http://search.proquest.com/docview/ 1288999103? accountid=11644\%5Cnhttp://sm2nn8xb3j.search. serialssolutions.com/?ctx_ver=Z39.88-2004\&ctx_enc=info:ofi/enc: UTF-8\&rfr_id=info:sid/PsycINFO\&rft_val_fmt=info:ofi/fmt:kev:mtx

3 World Health Organization. Non communicable diseases, 2015. Available: http://www.who.int/mediacentre/factsheets/fs355/en/

4 Rao DP, Orpana H, Krewski D. Physical activity and non-movement behaviours: their independent and combined associations with metabolic syndrome. Int J Behav Nutr Phys Act 2016;13:26.

5 Hill K, Gardiner PA, Cavalheri V, et al. Physical activity and sedentary behaviour: applying lessons to chronic obstructive pulmonary disease. Intern Med J 2015;45:474-82.

6 Hunter RF, Boeri M, Tully MA, et al. Addressing inequalities in physical activity participation: implications for public health policy and practice. Prev Med 2015;72:64-9.

7 Durstine JL, Gordon B, Wang Z, et al. Chronic disease and the link to physical activity. J Sport Health Sci 2013;2:3-11.

8 Jones SA, Wen F, Herring AH, et al. Correlates of US adult physical activity and sedentary behavior patterns. J Sci Med Sport 2016;19:1020-7.

9 Dumith SC, Hallal PC, Reis RS, et al. Worldwide prevalence of physical inactivity and its association with human development index in 76 countries. Prev Med 2011;53:24-8.

10 Rind E, Jones A. "I used to be as fit as a linnet" - beliefs, attitudes, and environmental supportiveness for physical activity in former mining areas in the North-East of England. Soc Sci Med 2015:126:110-8.

11 Loprinzi PD, Darnell T, Hager K, et al. Physical activity-related beliefs and discrepancies between beliefs and physical activity behavior for various chronic diseases. Physiol Behav 2015;151:577-82. 
12 Chaudhury H, Campo M, Michael Y, et al. Neighbourhood environment and physical activity in older adults. Soc Sci Med 2016;149:104-13.

13 Akpinar A. How is quality of urban green spaces associated with physical activity and health? Urban Forestry \& Urban Greening 2016;16:76-83.

14 Veitch J, Abbott G, Kaczynski AT, et al. Park availability and physica activity, TV time, and overweight and obesity among women: findings from Australia and the United States. Health Place 2016;38:96-102.

15 Cerin E, Sit CHP, Zhang CJP, et al. Neighbourhood environment, physical activity, quality of life and depressive symptoms in Hong Kong older adults: a protocol for an observational study. BMJ Open 2016;6:e010384.

16 Marquet O, Miralles-Guasch C. Neighbourhood vitality and physical activity among the elderly: the role of walkable environments on active ageing in Barcelona, Spain. Soc Sci Med 2015;135:24-30.

17 Coffee NT, Howard N, Paquet C, et al. Is walkability associated with a lower cardiometabolic risk? Health Place 2013;21:163-9.

18 a KK, a VR, English L, et al. Vigorous physical activity among college students: using the health belief model to assess involvement and social support. Arch Exerc Heal Dis 2014;4:267-79.

19 Rahmati-Najarkolaei F, Tavafian SS, Gholami Fesharaki M, et al. Factors predicting nutrition and physical activity behaviors due to cardiovascular disease in Tehran university students: application of health belief model. Iran Red Crescent Med J 2015;17:e18879.

20 Soleymanian A, Niknami S, Hajizadeh E, et al. Development and validation of a health belief model based instrument for measuring factors influencing exercise behaviors to prevent osteoporosis in premenopausal women (hope). BMC Musculoskelet Disord 2014;15:61.

21 Mahmood A, Chaudhury H, Michael YL, et al. A photovoice documentation of the role of neighborhood physical and social environments in older adults' physical activity in two metropolitan areas in North America. Soc Sci Med 2012;74:1180-92.

22 Brawner CA, Churilla JR, Keteyian SJ. Prevalence of physical activity is lower among individuals with chronic disease. Med Sci Sports Exerc 2016;48:1062-7.

23 Elmesmari R, Reilly JJ, Martin A, et al. Accelerometer measured levels of moderate-to-vigorous intensity physical activity and sedentary time in children and adolescents with chronic disease: a systematic review and meta-analysis. PLoS One 2017;12:e0179429-20.

24 Siddiqi A, Shahidi FV, Ramraj C, et al. Associations between race, discrimination and risk for chronic disease in a population-based sample from Canada. Soc Sci Med 2017;194:135-41.

25 Erikson EH. Identity and the life cycle: selected papers. London: Psychology Press, 1959.

26 Dennison L, Morrison L, Conway G, et al. Opportunities and challenges for smartphone applications in supporting health behavio change: qualitative study. J Med Internet Res 2013;15:e86-12.

27 Carlson JA, Remigio-Baker RA, Anderson CAM, et al. Walking mediates associations between neighborhood activity supportiveness and BMI in the Women's Health Initiative San Diego cohort. Health Place 2016;38:48-53.

28 MacDonald Gibson J, Rodriguez D, Dennerlein T, et al. Predicting urban design effects on physical activity and public health: a case study. Health Place 2015;35:79-84.

29 Sallis JF, Saelens BE. Assessment of physical activity by selfreport: status, limitations, and future directions. Res Q Exerc Sport 2000;71:1-14.

30 Sallis JF. Physical activity neighborhood environment survey (PANES), 2016. Available: http://sallis.ucsd.edu/measure_panes. htm

31 Hayslip B, Weigand D, Weinberg R, et al. The development of new scales for assessing health belief model constructs in adulthood. $J$ Aging Phys Act 1996;4:307-23.

32 International Physical Activity Questionnaire. IPAQ scoring protocol, 2016. Available: https://sites.google.com/site/theipaq/scoringprotocol

33 Sebastião E, Galvez PAE, Nakamura PM, et al. Activity behavior, nutritional status and perceived health in older Brazilian adults: does the number of chronic diseases matter? Geriatr Gerontol Int 2017;17:2376-82.

34 Zhou P, Hughes AK, Grady SC, et al. Physical activity and chronic diseases among older people in a mid-size City in China: a longitudinal investigation of bipolar effects. BMC Public Health 2018;18:1-16.

35 Paquet C, Coffee NT, Haren MT, et al. Food environment, walkability, and public open spaces are associated with incident development of cardio-metabolic risk factors in a biomedical cohort. Health Place 2014;28:173-6.

36 Ar-Yuwat S, Clark MJ, Hunter A, et al. Determinants of physical activity in primary school students using the health belief model. $J$ Multidiscip Healthc 2013;6:119-26.

37 Ribeiro Al, Mitchell R, Carvalho MS, et al. Physical activity-friendly neighbourhood among older adults from a medium size urban setting in southern Europe. Prev Med 2013;57:664-70.

38 Kaczynski AT, Mowen AJ. Does self-selection influence the relationship between Park availability and physical activity? Prev Med 2011;52:23-5.

39 Lovasi GS, Bader MDM, Quinn J, et al. Body mass index, safety hazards, and neighborhood attractiveness. Am J Prev Med 2012;43:378-84.

40 Gammage KL, Gasparotto J, Mack DE, et al. Gender differences in osteoporosis health beliefs and knowledge and their relation to vigorous physical activity in university students. J Am Coll Health 2012;60:58-64.

41 Tamirat A, Abebe L, Kirose G. Prediction of physical activity among type-2 diabetes patients attending Jimma university specialized Hospital, Southwest Ethiopia. Application of health belief mode 2014;2:524-31

42 Leischik R, Dworrak B, Strauss M. Plasticity of health. Ger J Med 2016;1:2-17. 